\title{
The Integrated Medicine route for women's Health and oncology in the Local Health Unit (AUSL) of Bologna and in the Emilia-Romagna Region
}

\author{
Grazia Lesi ${ }^{* 1}$ and Stefania Florindi ${ }^{2}$ \\ ${ }^{1 *}$ Gynecologist, Traditional Chinese Medicine, Acupuncturist, person in charge for CAM integration in Women's health and Oncological patient, \\ Bologna Local Health Unit, Italy \\ 2Sociologist, researcher, narrative medicine research, Asclepiadi Service Onlus
}

${ }^{*}$ Corresponding author: Grazia Lesi, Gynecologist, Traditional Chinese Medicine, Acupuncturist, person in charge for CAM integration in Women's health and Oncological patient, Bologna Local Health Unit, Italy

Received: September 20, 2019; Accepted: September 30, 2019; Published: October 23, 2019;

\section{Short Communication}

In contemporary medicine hyperspecialistic and technological visions coexist with a widespread illiteracy about what is psychophysical wellbeing. People are looking for solutions that are able to take into account physical and mental illnesses as a whole. On the other hand, health professionals live the contradiction between the increase in scientific knowledge and the rigidity of the therapeutic intervention field. Among them the most common feeling is frustration, which comes out in fact from the feeling of being mechanicals of the body or of the brain rather than promoters or facilitators of human health.

This is particularly true in the field of women's health and facing oncological diseases, where physical health is intertwined with personal, familiar, psychological and social aspects.

The integration proposal in the Emilia-Romagna Region and in the Local Health Unit of Bologna arises from these two discomforts: on the one hand from the need to know the effectiveness and the applicability of alternative and Complementary Medicine (CAM), according to the clinical efficacy criteria, within the services of the Local Health Unit and, on the other hand, from the request of women taking part in self-help groups for breast-operated women, who sought relief from side effects of adjuvant drugs.

Within the Local Health Unit of Bologna, the process of CAM integration in oncology and in the field of women's health was born in 2004, together with the First Experimental Programme for the integration of CAM in the regional health system. Some study projects were proposed and carried out on women's issues and with high social and health relevance.

First of all a pilot project exploring the effectiveness of acupuncture in a small group of women subjected to adjuvant therapy after breast cancer [1].

Then a survey on the free clinic services of the City of Bologna was carried out. The focus of the study was the use of CAM in women aged between 45 and 65 years (one year of observation) and the results showed that $30 \%$ of women used CAM professionals and/or CAM remedies, alone or together with replacement therapy. The survey, designed in collaboration with the University of Sydney, revealed a hidden side of CAM use and a trend that is still confirmed in the clinical reality $[2,3]$.

Afterwards a pilot study about the use of a technique derived from acupuncture, the needle-injection in the acupuncture point SP 6 (Sanyinjiao) of Vitamin K1, in order to reduce menstrual pain in young women suffering from severe primary dysmenorrhea. At the beginning of menstrual pain, 19 young women (aged between 15 and 19 years) were treated. The method reduced pain (measured by VAS) by about $50 \%$ in acute and decreased the use of analgesic drugs; this result was kept reduced for about 4 months after the initial treatment [4].

In oncology, the results of the pilot project and of the AcCliMaT project have furthered the continuation of the works, throughout selection criteria that can be found in the Regional guidance document, which represents the theoretical basis and the reference methodological framework for the Third Regional Experimental Programme about CAM [1]. Starting from the criteria presented there, the choice of the study focus on the prevention / reduction / control of adverse events, related to non-physiological menopause(which represents a serious problem for the quality of life of women with breast cancer), has been privileged.

\section{AcCliMaT Study Project}

AcCliMaT is a pragmatic, randomized and controlled trial comparing acupuncture plus enhanced self-care versus enhanced selfcare alone. A total of 190 women with breast cancer were randomly assigned. The acupuncture group received 10 traditional acupuncture treatment sessions, according to a therapeutic protocol derived from a consensus among the acupuncturists of the project group. Within the acupuncture treatment group, there was a reduction of the primary outcome, Hot Flash Score (HSF) that measures the intensity and frequency of hot flashes, significantly lower than the control group (46\% difference between the two groups). The Menopause Quality of 
Life (MenQoL) questionnaire also showed a better quality of life in the group with acupuncture plus self-care; a result that tends to remain in the follow up.

The AcCliMaT study confirms what has been highlighted in literature: acupuncture represents a possibility of treating and improving the quality of life in post-intervention of women with breast cancer and the integration between acupuncture and self-care represents a further advantage for the reduction of hot flushes and for the control of the climacteric syndrome, improving the quality of life of women with breast cancer. This is the basis on which the project of Integrative Medicine in Oncology was set up [5].

\section{Integrated Medicine in Oncology in Emilia-Romagna Region (Med.I.O.R.E.R.)}

The Med.I.O.R.E.R project represents the link between the research phase and the construction of acupuncture services dedicated to women operated for breast cancer. It is a prospective, multicentre study evaluating a model of integration of CAM treatments in the oncology departments and services of the Healthcare Centres of the Emilia-Romagna Region and responds to the need, more and more frequently expressed by patients suffering from breast cancer, to reduce the side effects of chemo-radiotherapy and adjuvant therapy. The project aims at the construction of integrated medicine surgeries in which Traditional Acupuncture plus Self-care treatments will be offered. The Med.I.O.R.E.R. project involves the measurement of quantitative process indicators and the collection of qualitative data related to the satisfaction of both professionals and patients.

The Primary Outcomes: The Primary Outcomes are frequency of sending patients to the CAM surgery, effective use of the path by patients, compliance, average number of accesses per patient to the integrated medicine surgery and the number of patients included for each centre.

The Secondary Outcomes: The Secondary Outcomes are the level of integration of the Acupuncture plus Self-care intervention in the clinical oncological practice of the Healthcare Centres of the Emilia-Romagna Region, dedicated to the climacteric syndrome in women with breast cancer; the adequacy of the offer according to the demand (external pressure index); the adequacy of the integrated medical pathway within the organization (focus groups, interviews of integrated medicine).

\section{Conclusion}

The Integrated Medicine route in oncology goes on with great belief and satisfaction from professionals and women with breast cancer and demonstrates how the inclusion of CAM methods, supported by scientific evidence and local clinical experiences conducted with scientific criteria, represents an advantage for the quality of life of women with breast cancer. At the same time, it confirms what Zhang Xiaorui (WHO Coordination Officer) wrote on December 2000: "The scientific, safe and effective use of traditional medicine will certainly further promote the development of traditional medicine and traditional medicine will undoubtedly make more and more contributions to human health in the 21st century".

\section{References}

1. http://assr.regione.emilia-romagna.it/it/funzioni/mnc/doc-omncer

2. Cardini F, LesiLombardoF,Van der Sluijs C (2010)The use of complementary and alternative medicine by women experiencing menopausal symptoms in Bologna. BMC Women's Health 10: 7.

3. Corinnevander Sluijs, Flavia L. Lombardo, GraziaLesi, Alan Bensoussan, Francesco Cardini (2013) Social and Cultural Factors Affecting Complementary and Alternative Medicine (CAM) Use during Menopause in Sydney and Bologna - Hindawi Publishing Corporation Evidence-Based Complementary and AlternativeMedicine 2013.

4. Grazia Lesi, Annagiulia Gramenzi, Clarissa Frascà, Francesco Cardini, Clede Maria (2017) Acupuncture Point Injection of Vitamin K1 to Treat Severe Primary Dysmenorrhea: Case Series at a Women's Health Service in Bologna. Garavini Chinese Medicine 8: 33-41.

5. Grazia Lesi, GiorgiaRazzini, Muriel Assunta Musti, Elisa Stivanello, Chiara Petrucci, et al. Acupuncture As an Integrative Approach for the Treatment ofHot Flashes in Women With Breast Cancer: A ProspectiveMulticenter Randomized Controlled Trial (AcCliMaT) J Clin Oncol 4: 1795-802.
Citation:

Lesi G and Florindi S (2019) The Integrated Medicine route for women's Health and oncology in the Local Health Unit (AUSL) of Bologna and in the Emilia-Romagna Region. Integr Gyn Obstet J Volume 2(4): 1-2. 